https://artnodes.uoc.edu

\title{
Entre la imaginación comprensiva y la imaginación compasiva. Apuntes para una pedagogía
}

\author{
Belén Altuna \\ Universidad del País Vasco (UPV/EHU) \\ Fecha de presentación: octubre de 2021 \\ Fecha de aceptación: diciembre de 2021 \\ Fecha de publicación: enero de 2022
}

\section{Cita recomendada}

Altuna, Belén. 2022. «Entre la imaginación comprensiva y la imaginación compasiva. Apuntes para una pedagogía». En: Garcés, Marina (coord.). «Ecología de la imaginación». Artnodes, no. 29. UOC. [Fecha de consulta: dd/mm/aa]. https://doi.org/10.7238/artnodes.v0i29.393021

cc Los textos publicados en esta revista están sujetos -si no se indica lo contrario- a una licencia de Reconocimiento 4.0 Internacional de Creative Commons. La licencia completa se puede consultar en https://creativecommons. org/licenses/by/4.0/deed.es

\section{Resumen}

El texto se pregunta por las posibles funciones de la imaginación en el ámbito ético-político y argumenta a favor del fomento pedagógico de dos formas de imaginación distintas, aunque fuertemente relacionadas: la imaginación comprensiva, que implica desarrollar «un modo de pensar amplio», como lo caracteriza Hannah Arendt, y la imaginación compasiva, que supone una potenciación no solo de los aspectos cognitivos de esta facultad, sino también de los afectivos y empáticos, como subraya Martin Hoffman. Finalmente, se repasan varios autores y tradiciones pedagógicas que han impulsado alguna de esas formas de imaginación 0, aún mejor, la combinación de ambas. Hablamos de entrenamientos educativos que tienen como objetivo ensanchar la mente y cultivar la humanidad, que ayuden a formar ciudadanos críticos y comprometidos con la búsqueda de formas más justas y equitativas de convivencia.

\section{Palabras clave}

imaginación; compasión; adopción de perspectiva; representación mental 


\section{artnodes}

\section{Between comprehensive imagination and compassionate imagination. Notes for a pedagogy}

\section{Abstract}

The text asks about the possible functions of imagination in the ethical-political sphere and argues in favour of the pedagogical promotion of two different, albeit strongly related, forms of imagination: the comprehensive imagination, which involves developing "a broad way of thinking", as Hannah Arendt characterises it, and the compassionate imagination, which involves an enhancement of not only the cognitive aspects of this faculty but also of the affective and empathic ones, as Martin Hoffman stresses. Finally, we review some authors and pedagogical traditions that have promoted one of these forms of imagination or, even better, a combination of both. We are talking about educational training that aims to broaden the mind and cultivate humanity, helping to form critical citizens committed to the search for fairer and more equitable forms of coexistence.

\section{Keywords}

imagination, sympathy, perspective-taking, mental representation 
Las siguientes páginas se suman a la invitación, en este número monográfico dedicado a las «Ecologías de la imaginación», a pensar la imaginación como una facultad ética, política y social, y a reflexionar en torno a cómo podríamos incentivar sus poderes ante los múltiples y acuciantes retos que se nos presentan. Como el campo es vastísimo y breve el espacio, habremos de conformarnos con esbozar algunas ideas sustanciales. En un primer capítulo, presentaremos las que podrían ser, a nuestro juicio, las principales funciones de la imaginación en este ámbito. En el segundo, nos centraremos en la imaginación comprensiva ( $\mathrm{y}$, en la medida de lo posible, comprehensiva), de la mano de Hannah Arendt. En el tercero, nos acercaremos en cambio a la imaginación compasiva, más afectiva que cognitiva, con la guía de Martin Hoffman. Finalmente, en el cuarto, presentaremos -aunque sea de forma breve- algunos acercamientos pedagógicos que buscan fomentar una u otra, o mejor, la combinación de ambas formas de imaginación, para la educación de una ciudadanía con una capacidad ensanchada de juicio ético y político, dilatada y afinada por esa mirada afectiva y cognitiva.

\section{Funciones de la imaginación en el ámbito ético, político y social}

Entendemos por imaginación un tipo de representación mental que, generalmente, solemos diferenciar de la mera creación o ensoñación fantasiosa. Mientras que la fantasía nos aleja de la realidad, a veces de manera lúdica y fabulosa, a veces de manera terrible y patológica, la imaginación es esa forma de representación que utilizamos para pensar, entender 0 transformar mejor esa misma realidad, no para huir de ella. Aunque sus funciones pueden ser múltiples, creo que podemos resaltar tres especialmente significativas para esos usos éticos, políticos y sociales que buscamos. Estas serían las funciones de la imaginación que más nos interesan:

\subsection{Representar y comparar las posibilidades de acción -individual y colectiva}

Podemos entender en gran parte la imaginación como una representación de posibilidades. Aunque pueda utilizarse también para replantear el pasado ("qué hubiera pasado si»), implica sobre todo concebir posibles presentes y futuros alternativos. Todo intento de trasformación social o política (además de la personal, por supuesto) puede ser fecundado por una rica variedad de proyecciones imaginativas que nos hagan probar mentalmente nuevos escenarios. Probar y evaluar las consecuencias previsibles de cada uno de ellos. Porque el principio de responsabilidad sería impracticable - 0 malamente practicable- sin esta capacidad de anticipar, ensayar y valorar las mejores formas y modalidades de acción ante una situación dada.

\subsection{Representar y ver tal elemento de una situación como otra cosa}

Sabemos que nuestro pensamiento funciona a base de esquemas, metáforas, narrativas; tenemos una gran capacidad para ver algo como otra cosa, para enmarcarlo o reencuadrarlo de forma imaginativa. A veces de manera sorprendentemente nueva, claro, y es ese aspecto creativo de la imaginación, su función poética 0 artística, la que ha sido ensalzada tantas veces. Pero no hace falta llegar a eso, porque toda nuestra percepción tiene que ver con cómo «encuadramos» una situación. Pongamos que esta hamburguesa que tengo en el plato está riquísima, que ha sido un gran acierto venir a este restaurante; tenían razón: el chef es excelente, un maestro en el uso de especias. Percibo la hamburguesa como un plato culinario. Pongamos en cambio que otra persona recuerda que esa misma hamburguesa viene de una ternera, que recrea e imagina el sufrimiento, el encierro y la matanza de ese animal, la forma en que ha sido gestado, criado y utilizado para el placer de unos cuantos humanos. Percibe la hamburguesa como un animal muerto, sacrificado. De la misma manera, podemos enmarcar y trasladar en nuestra mente a todos los seres que estemos considerando, 0 bien alejándolos 0 bien acercándolos a la esfera en que son permeables a nuestra empatía.

\subsection{Representar y adoptar el punto de vista de otra persona}

Es a esta función a la que vamos a dar un protagonismo especial, aunque necesariamente secundada y complementada por las anteriores. Empecemos por aclarar que esta capacidad de ponernos en el lugar de otra persona no es necesariamente empatía, aunque muchas veces se confunda con ella. Los psicólogos cognitivos y sociales prefieren emplear un término más específico, como es la adopción imaginativa de la perspectiva o del rol del otro (perspective-taking o role-taking): ${ }^{2}$ en primer lugar, una habilidad cognitiva imprescindible para las interacciones sociales, que nos hace conscientes de que la misma situación es y puede ser percibida e interpretada de manera diversa por la pluralidad de personas implicadas. La adopción de perspectiva meramente cognitiva suele denominarse fría a menudo, mientras que la empatía es (en su forma más compleja) una adopción de perspectiva caliente, en la

1. Reformulo y amplío aquí los tres modos principales de «percepción moral imaginativa» (adopción de perspectiva, (re)encuadre y comparación), que distingue y estudia Gibert (2014). He tratado ya esta distinción y las potencialidades éticas de la imaginación en otro lugar: Altuna (2018a), y sobre la relación entre imaginación, moral y empatía: Altuna (2018b).

2. Desde que G. H. Mead acuñara el concepto de role-taking, la distinción se ha utilizado a menudo. Véase, por ejemplo, Kohlberg (1992, 2009), Davis (1996) o Hoffman (2002). Para una taxonomía de los numerosos usos de empatía, véase Batson (2011). 
que las emociones siempre están implicadas y que tiene una dimensión afectiva, además de la cognitiva. La empatía implica así «compartir la emoción percibida en otro, sentir con otros (Eisenberg/Strayer 1992, 15), esto es, sentir una emoción congruente con la percibida o imaginada en el otro; para empezar, algún grado de contento por su alegría, algún grado de pena o dolor por su sufrimiento.

Estas tres funciones (que a menudo, cómo no, se solapan o entrelazan) pueden ser utilizadas de múltiples maneras, pero vamos a ver con un poco más de detenimiento qué puede implicar ensanchar nuestra imaginación comprensiva y qué nuestra imaginación compasiva.

\section{La imaginación comprensiva: «entrenar la imaginación para ir de visita”}

Cuando a Hannah Arendt le preguntaban por su pensamiento, respondía: básicamente, estoy interesada en comprender. Es decir, reconocía que el actuar, la actividad política o el activismo cívico son, por supuesto, fundamentales, pero insistía en que ella estaba interesada ante todo en comprender. Y es que ninguna praxis puede tener bases sólidas sin un constante esfuerzo por ampliar nuestro conocimiento y nuestra comprensión de los hechos y de las posibilidades que se nos abren. Sin procurar tener, sostendrá, una «mentalidad amplia».

Pues bien, Arendt ve en la imaginación la facultad que nos habilita para esa labor:

«Sólo la imaginación nos permite ver las cosas con su verdadero aspecto, poner aquello que está demasiado cerca de una determinada distancia de tal forma que podamos verlo y comprenderlo sin parcialidad ni prejuicio, colmar el abismo que nos separa de aquello que está demasiado lejos y verlo como si nos fuera familiar. Esta «distanciación» de algunas cosas y este tender puentes hacia otras, forma parte del diálogo establecido por la comprensión con ellas; la sola experiencia instaura un contacto demasiado estrecho y el puro conocimiento erige barreras artificiales (...). Sin este tipo de imaginación, que en realidad es la comprensión, no seríamos capaces de orientarnos en el mundo. Es la única brújula interna de la que disponemos» (Arendt 1998, 45).

Aquí Arendt invoca el evidente valor epistémico de la imaginación, esa «brújula interna», pero todavía no le otorga una clara función ética o política. A lo largo de su obra, sin embargo, enlazará esta idea con «el modo de pensar amplio» que ya tematizara Kant, es decir, con esa forma de pensar que consiste en ponerse en el lugar de cualquier otro, de representarnos y examinar las perspectivas de otras personas. Arendt relaciona la facultad del juicio ético y político con ese modo de pensar, y sentencia: «Pensar con una mentalidad amplia quiere decir que se entrena la propia imaginación para ir de visita» (2003b, 84). Es decir, la imaginación nos permite tener presentes a los ausentes, re-presentar en el escenario de nuestra mente las opiniones, las ideas, las preferencias de los otros, de los otros reales o de los otros hipotéticos; nos permite ir de una perspectiva a otra (de visita), ver una situación desde toda clase de puntos de vista antagónicos, «hasta que por fin se eleva desde esas particularidades hacia alguna generalidad imparcial» (2003a, 370).

Estamos hablando de una labor múltiple de adopción de perspectivas, pero ante todo en un sentido cognitivo, no tanto empático, como recalcará la propia Arendt. Porque de la misma manera que comprender el comportamiento de otros no es perdonarlo o justificarlo (sin ir más lejos, su ingente investigación en Los orígenes del totalitarismo 0 su famosa concepción de «la banalidad del mal» son intentos de comprensión, en absoluto de justificación), la forma de pensar amplia que requiere el pensamiento político crítico implica ejercer la capacidad de juzgar, pero de juzgar bien. En sus propias palabras:

«El pensamiento político es representativo; me formo una opinión tras considerar determinado tema desde diversos puntos de vista, recordando los criterios de los que están ausentes; es decir, los represento. Este proceso de representación no implica adoptar ciegamente los puntos de vista reales de los que se sustentan otros criterios y, por tanto, miran hacia el mundo desde una perspectiva diferente; no se trata de empatía, como si yo intentara ser o sentir como alguna otra persona, ni de contar cabezas y unirse a la mayoría, sino de ser y pensar dentro de mi propia identidad tal como en realidad no soy. Cuantos más puntos de vista diversos tenga yo presentes cuando estoy valorando determinado asunto, y cuanto mejor pueda imaginarme cómo sentiría y pensaría si estuviera en el lugar de otros, tanto más fuerte será mi capacidad de pensamiento representativo y más válidas mis conclusiones, mi opinión» (Arendt 2003a, 369; cursiva de la autora del artículo).

La validez de los juicios derivados de la práctica de ese pensamiento amplio «no sería objetiva y universal ni subjetiva, dependiente del capricho personal, sino intersubjetiva o representativa» $(1998,146)$, apuntará. Y es la imaginación la facultad que permite ese ensanchamiento, la capacidad de re-presentar diferentes puntos de vista, ver un mismo problema desde diversas perspectivas y ponerse en el lugar de los distintos implicados.

\section{La imaginación compasiva. Cognición caliente y empatía}

Las principales teorías sobre la educación y el desarrollo del juicio moral -del niño al adulto- que se han elaborado a lo largo del último siglo responden en gran medida al patrón señalado por Arendt. Así lo hace la más citada, la teoría cognitivo-evolutiva de Kohlberg (1992), que pone el acento en cómo se va construyendo nuestro razonamiento o nuestro juicio moral sobre lo que es justo. Pues bien, la etapa más avanzada de ese desarrollo -a la que muy poca gente accede- tendría que ver con esa capacidad de una toma de rol múltiple, de manera que podamos ponernos imaginariamente en el lugar de todas las personas implicadas en un conflicto dado, comprender tan diversos puntos de vista e intentar llegar a un acuerdo que satisfaga lo mejor posible las necesidades y los intereses legítimos de todos (una teoría que corre 


\section{artnodes}

https://artnodes.uoc.edu

Entre la imaginación comprensiva y la imaginación compasiva. Apuntes para una pedagogía

paralela a la ética del discurso que elaboran, desde la filosofía, Jürgen Habermas o K. 0. Apel).

Esta amplia tradición que prioriza los procesos racionales ha encontrado respuesta en otros psicólogos del desarrollo que le echan en cara, precisamente, su descuido de los factores afectivos. La capacidad para adoptar el punto de vista de otro, incluso la facilidad cognitiva para esa «mentalidad amplia», puede ser usada más bien para los propios intereses, para manipular mejor a esos sujetos o para beneficiarse de ese conocimiento. Como pregunta Martin Hoffman, uno de los principales psicólogos críticos con esa línea, «ipor qué ponerse en el lugar de otro es más útil cuando se persigue un fin prosocial que uno egoísta?» $(2002,117)$. Más aún, ¿cómo se hace para que, ante un conflicto dado, no solo tengamos una capacidad de des-centrarnos y salir de nuestro propio punto de vista para atender el de los otros, sino que, además, nos fijemos y atendamos especialmente a las víctimas, a los más desfavorecidos, a los más perjudicados? ¿Cómo, sobre todo, cuando estas víctimas no tienen voz, visibilidad, no están en el centro del interés público?

Ha sido precisamente Hoffman (2002) quien ha elaborado la más completa teoría del desarrollo moral prosocial basada en el papel de la empatía, enlazando fuertemente los aspectos cognitivos con los afectivos. Porque se entiende que la empatía puede dar lugar a distintos sentimientos morales. Que la atención empática ante una persona que sufre, pongamos, puede traducirse en compasión, por citar la principal de esas emociones y que a veces engloba de manera simbólica todas las demás (y de esa manera estamos utilizando la expresión imaginación compasiva). Pero también puede devenir en ira empática contra el causante de ese sufrimiento, si lo hay, o en indignación moral, si lo percibimos como un dolor injustamente infligido. De la misma manera, puede provocar un sentimiento de responsabilidad o culpa empática ante esa persona, si por acción o inacción hemos contribuido a su dolor.

En su estudio, Hoffman explora las formas en que los educadores pueden ayudar a madurar la tendencia empática inicial, intuitiva y preverbal, que en mayor o menor medida muestran casi todos los infantes. Y entre otros factores destaca el uso de la denominada «disciplina inductiva», que es la forma en que los padres u otros adultos pueden orientar al niño 0 a la niña sobre todo en los casos en que hacen daño a otros. Invitándoles, precisamente, a adoptar la perspectiva ajena y destacando el malestar producido por su acción. «No le grites que le haces llorar, ¿no ves que es muy pequeña?», «Está triste porque no le dejas jugar: tú ya has tenido tu turno, ahora le toca a él», «No le eches arena en la cara, que no le gusta; a ti tampoco te gustaría, ¿a que no?». Los padres también pueden sugerir actos de desagravio: «¿Por qué no le pides perdón a tu hermana e intentas que se sienta mejor?». De este modo, esta clase de disciplina saca partido «de la tendencia empática del niño, induciéndole a sentir aflicción empática y a sentirse culpable, darse cuenta del daño que su comportamiento puede hacer a otro y compararlo con sus deseos» $(2002,127)$.

Así entendida, la educación sentimental de los jóvenes ha de empezar muy pronto, no solo cuando ellos mismos transgreden alguna norma de convivencia 0 dañan a otros, sino también a través de sus experiencias como espectadores 0 conocedores del sufrimiento 0 de la victimización de otros. La hipótesis que desarrolla Hoffman es que «a los principios morales abstractos, aprendidos en contextos didácticos en frío (lecciones, sermones...), les falta fuerza motivacional. La contribución de la empatía a dichos principios es que los convierte en cogniciones prosociales cálidas (representaciones cognitivas llenas de afecto empático que les dan por tanto fuerza afectiva)» $(2002,201)$. Hay numerosas formas de impulsar esa educación moral sentimental desde el currículum escolar y proyectos pedagógicos que ensayan diferentes vías. Todos tienen, sin embargo, un aire de familia. No basta con enseñar datos, cifras, explicaciones científicas o históricas frías. Por supuesto que todo ello es imprescindible, pero cuando mejor se comprenden las historias sociales, no solo cognitiva sino también afectiva y empáticamente, es cuando se encarnan en una 0 varias víctimas concretas, cuando adquieren cara y nombre, y una narración que nos incita a ponernos en su piel, en sus circunstancias concretas. Esos ejemplos, esas experiencias, se guardan en la memoria como cogniciones cálidas, que se activan 0 actualizan cuando vemos, nos cuentan o leemos sobre una situación parecida.

Una de las principales críticas que suele dirigirse a la empatía es que peca de parcialidad. No es ningún secreto que nos es mucho más fácil empatizar 0 adoptar la perspectiva de la gente que nos es querida o familiar, de la gente que percibimos como similar a nosotros, 0 de la que está cerca, cara a cara, por ejemplo. En absoluto es tan fácil cuando esos otros son percibidos como extraños, adversarios 0 enemigos, 0 están simplemente diluidos en la gran masa indiferente. Por ello, una de las principales misiones de la educación moral consiste en superar la parcialidad empática por familiaridad y extenderla a otros grupos. Es la forma de luchar también contra todo tipo de prejuicios y estereotipos. Extendiendo la percepción de la semejanza con esos otros estigmatizados; individualizando, dando rostro, nombre, relato -a ser posible en primera persona- a alguno de ellos. Potenciando preferentemente una imaginación centrada en esa persona (¿te imaginas por lo que él/ella está pasando?), antes que una autocentrada (¿te imaginas cómo te sentirías tú si estuvieras en esa situación?), aunque tal vez la más efectiva sea una combinación de ambas. 0 invitando a imaginar que esos hechos le sucediesen a alguien muy cercano y querido: ¿y si le hubiesen hecho eso a tu hermana, y si ese hombre fuera tu padre? Lo fascinante es que en esos casos usamos la parcialidad empática por familiaridad volviéndola contra sí misma, poniéndola al servicio del desarrollo motivacional prosocial, como insiste Hoffman. Fomentando, en definitiva, una imaginación compasiva.

\section{Combinaciones de ambas: las pedagogías de la imaginación}

Una parte importante de la educación consiste 0 debería consistir, por tanto, en ejercicios para ponernos en el lugar de los otros; para 


\section{artnodes}

https://artnodes.uoc.edu

Entre la imaginación comprensiva y la imaginación compasiva. Apuntes para una pedagogía

imaginar escenarios pasados, presentes o futuros, reales o meramente hipotéticos; para comprender, relacionar y ensanchar el campo de las posibilidades humanas. Algunas tradiciones pedagógicas han insistido en los aspectos cognitivos de esa ampliación; otras, en los éticos y afectivos. Lo que sigue es tan solo una pequeña muestra de esa riqueza.

En la tradición budista, por ejemplo, existen numerosas propuestas pedagógicas para meditar e incentivar la compasión por todos los seres. Karen Armstrong nos relata una de ellas, un ejercicio que ayuda a apreciar hasta qué punto somos dependientes de tantas y tantas personas que no conocemos y que pueden vivir muy lejos de nosotros: «Cuando pasees alrededor de tu casa, recuerda a todas las personas que la construyeron, trataron la madera, cocieron los ladrillos, instalaron las tuberías y tejieron tu ropa blanca. Cuando te levantes por la mañana, recuerda a quienes plantaron, recogieron e hilaron el algodón de tus sábanas, y también a quienes cosecharon, trataron y exportaron los granos que mueles para tu café matinal. Disfrutas de su producto, de modo que tienes una responsabilidad hacia ellos, especialmente si trabajaban en condiciones miserables. ¿Quién coció el pan que tomas para desayunar? Piensa en el esfuerzo que supuso la producción de cada rebanada. Cuando te dirijas al trabajo, reflexiona sobre los miles de trabajadores e ingenieros que construyeron las carreteras, los coches, las líneas de ferrocarril, los aviones, los trenes y el metro de los que dependes. Continúa con este ejercicio durante todo el día... Somos lo que somos debido al trabajo duro, las ideas y los logros de una cantidad innumerable de personas» (Armstrong 2011, 137).

En la tradición pedagógica occidental, tenemos la labor seminal de Rousseau. Recordemos el empeño que pone en enseñar a su Emilio a excitar y nutrir su sensibilidad naciente, en presentarle ocasiones para guiar su empatía y su compasión, mostrándole la semejanza entre su sufrimiento y el de los demás, cualesquiera que estos sean. También en su insistencia para que sea consciente de las vicisitudes de la fortuna y para que no olvide la contingencia de su condición: «No acostumbréis a vuestro alumno a mirar desde lo alto de su gloria las penas de los infortunados, los trabajos de los miserables, ni esperéis enseñarle a compadecerlos si los considera como extraños a él. Hacedle comprender bien que la suerte de esos desgraciados puede ser la suya» (1998, 332).

A lo largo de la obra, el maestro Rousseau no ceja en su empeño en despertar la imaginación moral de su discípulo. Relata, por ejemplo, una ocasión en la que ambos van a comer a una casa opulenta. Se encuentran con los preparativos de un festín, con mucha gente, muchos criados, muchos platos. «Todo este aparato de placer y de fiesta tiene algo de embriagador que se sube a la cabeza cuando no estamos acostumbrados», cuenta. Así, para que su alumno adolescente no se deje llevar sin más y piense, se acerca a su oído y le dice: «¿Por cuántas manos pensáis que ha pasado todo lo que veis sobre esta mesa antes de llegar aquí?» $(1998,280) .{ }^{3}$ Lo incita, así, a completar la percepción directa del lujo del festín con la percepción indirecta, mediatizada por la reflexión y la imaginación, del origen y el recorrido de esos bienes. Lo anima, en consecuencia, a tener un punto de vista ampliado sobre la escena, conjugándolo con los conocimientos políticos y sociales previos; a tener presentes, en suma, a los numerosos trabajadores que con su esfuerzo invisible han hecho posible el festín, y tal vez a plantearse la justicia o injusticia de su situación.

No es casual que encontremos ejercicios de ese estilo en grandes pedagogos de los siglos XIX-XX, como es el caso de John Dewey (2011). En su propuesta didáctica, el aula se transforma en una prolongación del mundo real, de manera que se prepara a los alumnos para ser ciudadanos activos que realizan proyectos en común con sus compañeros. Así, las clases se inician con una actividad práctica, como cocinar, tejer 0 hacer labores de jardinería. A partir de ahí, se les proponen distintos problemas y cuestiones. Por ejemplo, "¿¿de dónde provienen estos materiales?”, “¿quién los creó”, “¿mediante qué formas de trabajo llegaron a mí?", “¿cómo podemos imaginar la organización social de esas formas de trabajo?"». ${ }^{4}$ Dewey muestra cuántas ideas complejas pueden derivarse de una tarea en apariencia tan sencilla como hilar algodón, ideas que pueden ir en múltiples direcciones, históricas, económicas 0 científicas. Se puede preguntar por qué es tan difícil preparar el algodón para hacer tejidos, pero también cuál es la relación de esa dificultad práctica con el hecho de que durante tanto tiempo hayan sido esclavos quienes se encargaran de ese trabajo.

En ejercicios de este tipo encontramos la misma idea que llevó a C. Wright Mills a escribir, en 1959, La imaginación sociológica, donde la muestra como el gran instrumento de toda tarea sociológica, pero también como la capacidad que debería desarrollar cualquier persona -y desde luego, cualquier joven que se esté formando- que quisiera entender con un poco de amplitud lo que está ocurriendo en el mundo, sí, pero también cómo se integra su propia biografía en esa intersección entre la sociedad y la historia:

«Porque esa imaginación es la capacidad de pasar de una perspectiva a otra: de la política a la psicológica, del examen de una sola familia a la estimación comparativa de los presupuestos nacionales del mundo, de la escuela teológica al establecimiento militar, del estudio de la industria del petróleo al de la poesía contemporánea. Es la capacidad de pasar de las transformaciones más impersonales y remotas a las características más íntimas del yo humano, y de ver las relaciones entre ambas cosas. Detrás de su uso está siempre la necesidad de saber el significado social e histórico del individuo en la sociedad y el periodo en que tiene su cualidad y su ser» (Mills 2004, 27).

La forma en que lo caracteriza Wright Mills tiene que ver con percibir, relacionar y comprender, es decir, con virtudes cognitivas. Con esa «brújula interna» de la que hablaba Hannah Arendt, como una distanciación de algunas cosas y un tender puentes hacia otras, lo que

3. Chavel (2012) utiliza también esta escena para intentar comprender qué es una «percepción justa». En general, este libro de Chavel, como el de Gibert (2014), Coecklebergh (2007) y 0xley (2011), ofrecen visiones muy sugerentes de los usos éticos, políticos y sociales de la imaginación.

4. Nussbaum $(2010,98)$ relata con detalle el método de Dewey. 
«forma parte del diálogo establecido por la comprensión con ellas». Un entrenamiento de la imaginación para «ir de visita» a otras mentes y perspectivas, sí, pero también para relacionar las distintas dimensiones de la realidad.

Vemos, por tanto, que algunos autores acentúan la parte cognitiva de «ese modo de pensar amplio», mientras que otros subrayan las virtudes éticas ligadas a la potenciación de la imaginación compasiva. Martha Nussbaum es, seguramente, la pensadora contemporánea que con mayor pasión y elocuencia insiste en unir ambas vertientes. Lo vemos en su encendida defensa de las humanidades, a las que relaciona «con la búsqueda del pensamiento crítico y los desafíos a la imaginación, así como con la comprensión empática de una variedad de experiencias humanas y de la complejidad que caracteriza nuestro mundo» (2010, 26). Precisamente, educar es -0 debería ser-cultivar la humanidad que hay en nosotros, más allá de preparar a los jóvenes para un mercado laboral incierto, precario y competitivo. Por ello, recalca, la democracia necesita de las artes y las humanidades, necesita educar, racional y sentimentalmente, encendiendo tanto una imaginación comprensiva como compasiva, necesita formar ciudadanos críticos y comprometidos con la búsqueda de formas más justas y equitativas de convivencia.

Recordemos cuáles son las tres habilidades que, a juicio de Nussbaum, debería impulsar esa pedagogía:

1) en primer lugar, la habilidad para desarrollar lo que, siguiendo a Sócrates, podríamos llamar una vida examinada, es decir, la capacidad para analizarse críticamente a uno mismo y a las propias creencias y tradiciones;
2) además, la habilidad de verse a sí mismo no solo como un ciudadano perteneciente a alguna región o grupo, sino también y, sobre todo, como un ser humano vinculado a los demás seres humanos por lazos de reconocimiento y mutua preocupación, lo que supone la capacidad de «trascender las lealtades nacionales y de afrontar los problemas internacionales como "ciudadanos del mundo"»;

3) y, por último y no menos importante, la habilidad para la imaginación narrativa, que está estrechamente ligada a las anteriores y que implica «la capacidad de pensar cómo sería estar en el lugar de otra persona; ser un lector inteligente de la historia de esa persona, y comprender las emociones, deseos y anhelos que alguien así pudiera experimentar» (2001, 29).

En definitiva, estamos hablando de toda una serie de ejercicios de ampliación imaginativa que nos permitan dilatar nuestra capacidad de juicio ético y político. Que nos permitan desafiar, por ejemplo, nuestro habitual imaginario social etnocéntrico (anclado en nuestra experiencia nacional 0 , en último término, occidental), androcéntrico (que privilegia el punto de vista masculino) 0 antropocéntrico (que no toma en consideración al resto de los animales 0 al conjunto de la naturaleza). De ejercicios imaginativos que puedan apuntar a nuestros puntos ciegos, a las muchas injusticias que no vemos 0 hacia las que no somos sensibles, o frente a las cuales no sabemos cómo actuar. Si la educación «es una invitación a comprender el presente para poder imaginar el futuro. La invitación a hacer próximo lo extraño y extraño lo próximo» (Garcés $2020,167)$, no cabe duda de que ha de consistir, precisamente y en gran medida, en ese tipo de entrenamientos. 


\section{Referencias bibliográficas}

Altuna, Belén. «La imaginación moral, o la ética como actividad imaginativa». Daimon, Revista Internacional de Filosofía, no. $74\left(2018^{\mathrm{a}}\right)$.

Altuna, Belén. «Empatía y moralidad: las dimensiones psicológicas y filosóficas de una relación compleja». Revista de Filosofía, vol. 43, no. 2 (2018b). Universidad Complutense. DOl: https:// doi.org/10.5209/RESF.62029.

Arendt, Hannah. De la historia a la acción. Barcelona: Paidós, 1998.

Arendt, Hannah. Entre el pasado y el futuro. Barcelona: Península, 2003a.

Arendt, Hannah. Conferencia sobre la filosofía política de Kant. Barcelona: Paidós, 2003b.

Armstrong, Karen. Doce pasos hacia una vida compasiva. Barcelona: Paidós, 2011.

Batson, David. «These things called Empathy: eight related but distinct Phenomena». En: Decety \& Ickes (eds.). The Social Neuroscience of Empathy (2011). Massachusests Institute of Technology,

Chavel, Solange. Se mettre à la place d'autrui. L'imagination moral. Press Universitaires de Rennes, 2012.

Coeckelbergh, Mark. Imagination and Principles.AnEssayon the Role of Imagination in Moral Reasoning. Hampshire: Palgrave MacMillan, 2007. D0l: https://doi.org/10.1057/9780230589803.

Davis, Mark H. Empathy: a social psychological approach. Boulder: Westview Press, 1996.

Dewey, John. Democracy and Education: An Introduction to the Philosophy of Education. Simon \& Brown, 2011.
Eisenberg, Nanacy, Strayer, Janet. La empatía y su desarrollo. Bilbao: Desclée de Brower, 1992.

Fasmire, Steven. «Morality as Art: Dewey, Metaphor, and Moral Imagination». Transactions of the Charles S.Peirce Society, vol. 35, no. 3 (1999).

Garcés, Marina. Escuela de aprendices. Barcelona: Galaxia Gutenberg, 2020.

Gibert, Martin. L'imagination en morale. Herman, L'avocat du diable, 2014.

Hoffman, Martin L. Desarrollo moral y empatía. Implicaciones para la atención y la justicia. Barcelona: Idea Books, 2002.

Kohlberg, Lawrence. Psicología del desarrollo moral. Bilbao: Desclée de Brouwer, 1992.

Mills, Charles Wright. La imaginación sociológica. México: FCE, 2004.

Nussbaum, Martha. El cultivo de la humanidad. Una defensa clásica de la reforma en la educación liberal. Andrés Bello, 2001.

Nussbaum, Martha. Sin fines de lucro. Por qué la democracia necesita de las humanidades. Katz, 2010. D0I: https://doi. org/10.2307/j.ctvndv60c.

Oxley, Julinna. The moral dimensions of Empathy. Hampshire: Palgrave MacMillan, 2011. DOl: https://doi. org/10.1057/9780230347809.

Rousseau, Jean-Jacques. Emilio (1762). Madrid: Alianza, 1998.

Vetlesen, Arne Johan. Perception, Empathy and Judgement. Pennsylvania State University Press, 1994. DOl: https://doi. org/10.1515/9780271071794. 


\section{artnodes}

\section{CV}

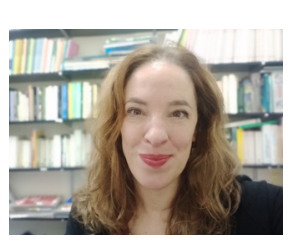

\section{Belén Altuna}

Facultad de Educación, Filosofía y Antropología

Universidad del País Vasco (UPV/EHU)

belen.altuna@ehu.eus

Doctora en Filosofía y profesora de Ética y Filosofía política en la Facultad de Educación, Filosofía y Antropología de la Universidad del País Vasco (UPV/EHU), en Donostia-San Sebastián. Entre sus publicaciones se encuentran los libros Euskaldun fededun. Euskaldun ona izateko modu baten historia (Alberdania, 2003; en castellano, El buen vasco. La tradición 'Euskaldun fededun', Hiria, 2012) y Una historia moral del rostro (Pre-Textos, 2010), así como numerosos artículos. Después de la ética y la antropología del rostro y de la alteridad desarrolladas en ese último libro, inspirada entre otros en el pensamiento de Emmanuel Lévinas y de Paul Ricoeur, sus investigaciones han ido dirigidas a profundizar en algunos de los mecanismos que operan en una verdadera «banalidad del mal»: los sesgos y límites de nuestra capacidad empática, las dificultades para ponernos en la piel del otro y las distintas formas de ceguera moral. Frente a ello, ha estudiado los modos en que podría impulsarse una imaginación ética a la altura de los desafíos de nuestro tiempo, ligada a un principio de responsabilidad necesariamente ampliado, como recogerá en un libro de próxima publicación. 